\title{
Evaluation and Analysis of the Satisfaction Degree of the Intelligent Outpatient Clinic of Tianjin 3A Grade Hospitals Based on the KANO Model
}

\author{
Fanyu Zhao, Yawen Xiao, Xue Feng, Chang Liu, He Jiang*
}

School of Nursing, Tianjin Medical University, Tianjin 300070, China

Email: zfy0129@126.com

\begin{abstract}
Objective - Based on the Kano model, we investigated the outpatients' satisfactions with the functions of the intelligent outpatient clinic of 3A Grade hospital in Tianjin, to improve the function of the intelligent outpatient clinic. Methods - Based on the Kano model, a questionnaire was designed to investigate the satisfaction and expectation of Tianjin outpatients with the use of intelligent outpatient clinic. A survey was conducted on 384 outpatients to determine the functions of the intelligent outpatient clinic. Results - Among the 14 intelligent outpatient service, $11(79 \%)$ are attractive quality, $3(21 \%)$ are one-dimensional quality, and there are no must-be quality. In the quadrant diagram, there are 3 requirements in the dominant area and 11 requirements in the retention area. There are three high satisfying attributes: convenience of multifunctional payment method, convenience of reservation registration and security of multiple payment methods. There is an unsatisfying function: the information pushed by WeChat platform. Conclusion - The intelligent outpatient clinic function has been satisfied by most patients, but it hasn't been fully popularized. It is necessary to improve the payment security, the order waiting and other attractive quality, to optimize the medical experience of the intelligent clinic and improve patients' satisfaction.
\end{abstract}

Keywords: intelligent outpatient clinic, KANO model, satisfaction

Against the background of "Healthy China 2030", The State Council attaches great importance to and continues to promote the construction of the "Internet + medical health" model to accelerate the integration of information technology and medical technology, aiming at providing people with high-quality, efficient and convenient medical and health services $^{[1]}$. Currently,the construction of smart clinics has become an integral part of the Internet in the process of the construction of hospital, most domestic level-3 medical institutions have been made with the use of the Internet to make an appointment, online payment and other intelligent outpatient service function ${ }^{[2]}$. However, the overall construction of smart outpatient service in China is still in the early stage, the overall utilization rate for patients is low, and the main function needs to be continuously improved ${ }^{[3]}$. These problems will affect the service efficiency and service quality of medical service supply. Kano model is a simple and feasible technical theory for identifying service attributes. Applying it to the evaluation of smart outpatient service can accurately identify the service needs of patients, determine different types of needs, and improve their deficiencies. Based on this, with the theoretical guidance of Kano model, this study investigated the status quo of smart outpatient service and the satisfaction of outpatient patients in third-grade A hospitals in six districts of Tianjin, classified 14 functional attributes of smart outpatient service, determined the type of demand, and provided targeted strategic suggestions for optimizing the function of smart outpatient service.

\section{Research object}

The convenience sampling method was used to select 384 outpatients from third-grade A hospitals in six districts of Tianjin in March 2019 as the study subjects. Inclusion criteria: (1) The ability to complete the questionnaire independently or those who can complete the questionnaire by assistance; (2) With clear consciousness; (3) He/She has been treated at least once in our hospital. Exclusion criteria: Unwillingness to participate in the study after explanation by the researcher.

\section{Research methods}

\subsection{Kano model}

The Kano model based analysis method is a model proposed by The Japanese scholar Noriaki Kano to classify and prioritize user needs on the basis of analyzing the impact of user needs on user satisfaction ${ }^{[4]}$. According to product, service quality and user satisfaction, Kano model divides service quality characteristics into 5 categories: Attractive Quality, 
One-dimensional Quality, Must-be Quality, Indifference Quality and Reverse Quality. Please see Figure 1.

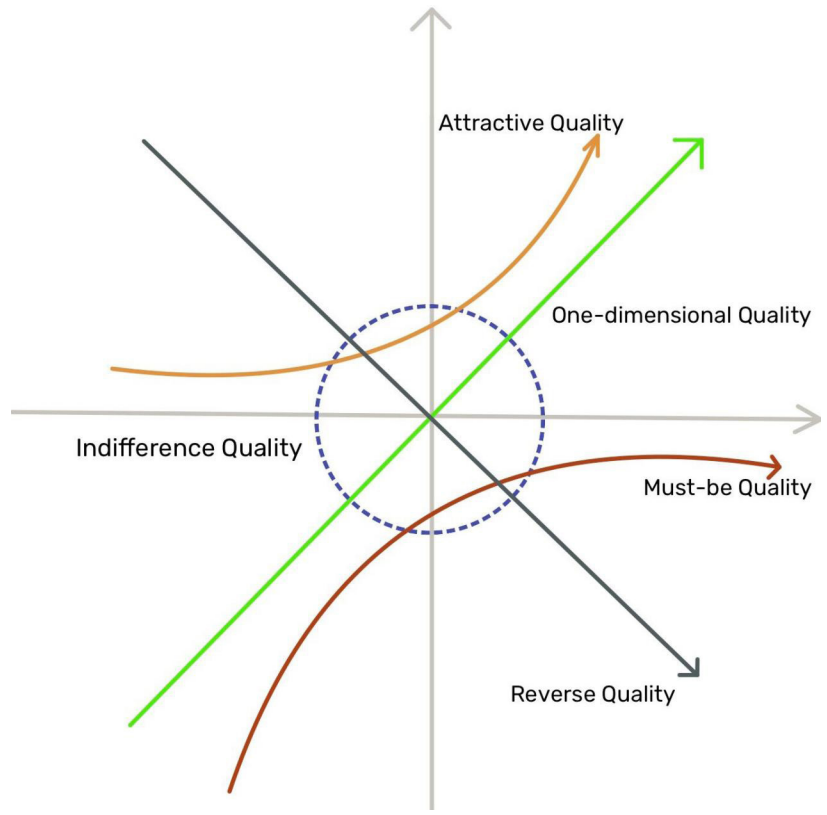

Figure 1. Kano model

\subsection{Survey tools}

According to the functions of smart clinics, this study developed a questionnaire based on Kano model, including three parts: basic information: gender, age, registered permanent residence and use of smart clinics. Satisfaction survey: A total of 14 items, Likert 5-level score was adopted from "very satisfied" to "very dissatisfied" according to the satisfaction degree, with a full score of 70 . The higher the score, the more satisfied the patients were with the function of smart clinics. Demand survey: It is compiled based on 7 main functions of smart clinics, including survey appointment registration, multi-functional self-service, intelligent guidance,calling for diagnosis, multiple payment methods, intelligent drug distribution and Wechat platform. According to the Kano model, the items of each function are designed into positive and negative aspects, and each patient can get $5 \times 5$ possible response combinations. The classification of Kano attributes is shown in Table 1. The pre-experimental results showed that the bartlett sphere test result was $\mathrm{P}<0.05$.

Table 1. Kano model requirements analysis evaluation table

\begin{tabular}{|c|c|c|c|c|c|}
\hline \multirow{2}{*}{ Product/service demand scale } & \multicolumn{5}{|c|}{ Negative questions } \\
\hline & Likes & It should be & Does not matter & Can be accepted & Dislikes \\
\hline \multicolumn{6}{|l|}{ Positive questions } \\
\hline Likes & Q & A & A & A & $\mathrm{O}$ \\
\hline It should be & $\mathrm{R}$ & I & I & I & M \\
\hline Does not matter & $\mathrm{R}$ & I & I & I & M \\
\hline Can be accepted & $\mathrm{R}$ & I & I & I & M \\
\hline Dislikes & $\mathrm{R}$ & $\mathrm{R}$ & $\mathrm{R}$ & $\mathrm{R}$ & Q \\
\hline
\end{tabular}

\subsection{Survey methods}

The members of the research group shall issue the questionnaire uniformly. Before the questionnaire is issued, they shall be trained to clarify the purpose and fill in the matters. The questionnaire shall be collected and checked on the spot, and any omission shall be pointed out and filled in timely. A total of 390 questionnaires were sent out in this survey, 384 of which were effectively received, with an effective response rate of $98.64 \%$. 


\subsection{Statistical methods}

EpiData 3.0 was used to input data and SPSS 20.0 was used for statistical analysis. The general data are described by means, standard deviation, frequency and percentage. According to the analysis method of Kano model, each functional attribute is determined, the Satisfaction Index (SI) and the Dissatisfaction Index (DSI) of each service Index are calculated, and the quadrant chart is made and analyzed.

\section{Results}

\subsection{Basic information}

Among 384 research objects, 181 (47.1\%) were males and 203 (52.9\%) were females. 126 (32.8\%) aged 18-29, $122(31.8 \%)$ aged 30-44, 80 (20.8\%) aged 45-60, and 56 (14.6\%) aged 61 and above. Besides,283 (73.7\%) were with registered permanence residence in Tianjin and 101 (26.3\%) were not registered in Tianjin. In the use of smart clinic, the top three most frequently used are appointment registration, online payment and waiting call number. Functions with low frequency of use are intelligent drug dispensing and wechat platform information push, as shown in Table 2.

Table 2. Basic data $(\mathbf{n}=\mathbf{3 8 4})$

\begin{tabular}{ccc}
\hline Issues & Number of people & Proportion (\%) \\
\hline Gender & 181 & 47.1 \\
Male & 203 & 52.9 \\
Female & & \\
Age & 126 & 32.8 \\
18-29 & 122 & 31.8 \\
$30-44$ & 80 & 20.8 \\
$45-60$ & 56 & 14.6 \\
Above 61 & & \\
Registered permanent residence & 283 & 73.7 \\
In Tianjin & 101 & 26.3 \\
Outside Tianjin & & \\
Use of smart clinics & 371 & 10.7 \\
Appointment register & 266 & 96.6 \\
Multiple payment methods & 243 & 69.3 \\
Calling for diagnosis & 195 & 63.3 \\
Multi-function self-service & 41 & 50.8 \\
Smart guidance & 31 & 1.6 \\
\hline Wechat platform for imformation push & & \\
\hline distribution of medications & & \\
\hline
\end{tabular}

\subsection{Scores of patient satisfaction for each function in smart clinic}

The results show that the highest score of satisfaction evaluation is the payment function, which is the convenience $(4.58 \pm 0.69)$ and security $(4.52 \pm 0.71)$ of the multi-function payment method. Wechat platform is proved with low

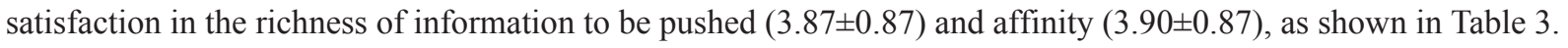

\subsection{KANO attribute analysis results of each function in smart clinics}

By calculating the frequency of the corresponding attribute of each function of the smart clinics, the functional attribute corresponding to the maximum frequency is the corresponding attribute of the service item considered by patients. The results showed that attractive quality accounted for 79\% (11 items) of the total number of functional items, while one-dimensional quality accounted for only $21 \%$ (3 items). It can be seen from Table 3 that the item with the highest SI value is "time accuracy of appointment registration" (0.755), indicating that patient satisfaction will be increased by $75.5 \%$ after improving the time accuracy of appointment registration, while the item with the highest DSI value is "security of multiple payment methods" (0.671), indicating that if this service is ignored, patient satisfaction will decrease by $67.1 \%$. 
Table 3. Kano quality analysis results of non-medical technical services in smart clinics $(\mathbf{n}=\mathbf{3 8 4})$

\begin{tabular}{|c|c|c|c|c|c|c|c|c|c|c|}
\hline \multirow{2}{*}{ Major function } & \multicolumn{6}{|c|}{ The classification basis of each attribute mark element(\%) } & \multirow{2}{*}{$\begin{array}{l}\text { Attribute } \\
\text { classification }\end{array}$} & \multirow{2}{*}{$\begin{array}{c}\text { Satisfaction } \\
\text { scores }\end{array}$} & \multicolumn{2}{|c|}{$\begin{array}{l}\text { Coefficient of } \\
\text { satisfaction }\end{array}$} \\
\hline & A & $\mathrm{O}$ & M & I & $\mathrm{R}$ & Q & & & SI & DSI \\
\hline \multicolumn{11}{|l|}{ Online Registration } \\
\hline 1Time accuracy & 48.7 & 26.3 & 10.2 & 14.2 & 0 & 0.6 & A & 4. $15 \pm 0.70$ & 0.755 & 0.367 \\
\hline 2Covenience & 43.5 & 28.6 & 7. 0 & 20.3 & 0.3 & 0.3 & A & $4.53 \pm 0.75$ & 0.725 & 0.358 \\
\hline \multicolumn{11}{|l|}{ Multi-function self-service } \\
\hline 3Artificial guide & 48.6 & 18.2 & 8.6 & 23.9 & 0.3 & 0.3 & A & 4. $11 \pm 0.91$ & 0.673 & 0.270 \\
\hline 4Operational convenience & 46.8 & 25 & 7.1 & 20.9 & 0 & 0.3 & A & 4. $39 \pm 0.82$ & 0.719 & 0.322 \\
\hline \multicolumn{11}{|l|}{ Smart guidance } \\
\hline 5Accuracy & 38.8 & 29.2 & 11 & 20.6 & 0.3 & 0.3 & A & 4. $24 \pm 0.88$ & 0.683 & 0.404 \\
\hline 6Convenience & 47.9 & 24.2 & 6.0 & 21.6 & 0.3 & 0 & A & 4. $30 \pm 0.88$ & 0.723 & 0.303 \\
\hline \multicolumn{11}{|l|}{ Calling for diagnosis } \\
\hline 7Orferliness & 28.6 & 45.1 & 13.8 & 12.2 & 0 & 0.3 & $\mathrm{O}$ & $4.43 \pm 0.83$ & 0.739 & 0.591 \\
\hline 8Convenience & 43.2 & 31.2 & 8.9 & 16.4 & 0.3 & 0 & A & 4. $45 \pm 0.81$ & 0.746 & 0.402 \\
\hline \multicolumn{11}{|l|}{ Multiple way of payment } \\
\hline 9Safety & 22.4 & 47.4 & 19.8 & 10.5 & 0 & 0 & $\mathrm{O}$ & $4.52 \pm 0.71$ & 0.697 & 0.671 \\
\hline 10Conveniernce & 44.3 & 30.5 & 6.8 & 18.5 & 0 & 0 & A & 4. $58 \pm 0.69$ & 0.747 & 0.373 \\
\hline \multicolumn{11}{|l|}{$\begin{array}{l}\text { Smart distribution of } \\
\text { medications }\end{array}$} \\
\hline 11Accuracy & 31.2 & 35.7 & 16. 2 & 16.6 & 0 & 0.3 & $\mathrm{O}$ & 4. $19 \pm 0.83$ & 0.671 & 0.521 \\
\hline 12Convenience & 48.2 & 20.6 & 8.4 & 22.4 & 0.3 & 0.3 & A & 4. $18 \pm 0.84$ & 0.691 & 0.291 \\
\hline \multicolumn{11}{|l|}{$\begin{array}{l}\text { Information pushed by } \\
\text { wechat platform }\end{array}$} \\
\hline 13Richness & 45.9 & 11.7 & 2.6 & 39.4 & 0 & 0.5 & A & $3.87 \pm 0.87$ & 0.578 & 0.144 \\
\hline 14Affinity & 47.9 & 12 & 2.3 & 37.3 & 0.3 & 0.3 & A & $3.90 \pm 0.87$ & 0.602 & 0.144 \\
\hline
\end{tabular}

Note: $\mathrm{SI}=(\mathrm{A}+\mathrm{O}) /(\mathrm{A}+\mathrm{O}+\mathrm{M}+\mathrm{I})$; $\mathrm{DSI}=(\mathrm{M}+\mathrm{O}) /(\mathrm{A}+\mathrm{O}+\mathrm{M}+\mathrm{I})$; the closer $\mathrm{SI}$ is to 1 , the greater the impact of this item on patient satisfaction; the closer DSI is to 1 , the greater the influence of this item on patient dissatisfaction is.

\subsection{Analysis of Si-DSI quadrant diagram}

Taking SI as the abscissa and DSI as the ordinate to draw the quadrant diagram. The first, second, third and fourth quadrants are the advantage area, improvement area, observation area and retention area respectively. Smart clinic functions were 7,9,11 in the advantage area and 1,2,3,4,5,6,8,10,12,13,14 in the retention area. See Figure 2.

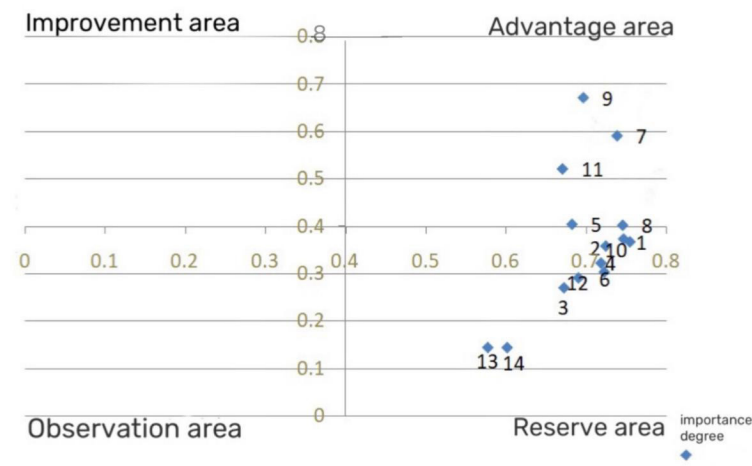

Figure 2. Quadrant summary of the results of satisfaction and importance of each function in smart clinic

Note: Advantage area: this area has high importance and satisfaction, so it needs to keep the advantage; Improvement area: This area has high importance but low satisfaction, which needs to be further strengthened; Observation area: The importance and satisfaction of this area are low, and this area does not need to be improved immediately; Reserved area: an area of low importance with high satisfaction, which can increase patient satisfaction and can still be retained but does not require much time. 


\section{Discussion}

\subsection{Analysis of patients' satisfaction with smart outpatient service}

Among the 7 functions of smart clinic, the usage rate and satisfaction score of payment function and appointment and registration function are at the top of the list. It can be seen that the payment function and appointment function of smart clinic are needed by patients and have been widely affirmed. Such functions should be guaranteed and improved first. However, the utilization rate and satisfaction score of wechat platform push information function are lower than other functions, indicating that the current hospital wechat platform is not used enough, such as the single push content, lack of communication recovery function and other problems. In today's information age, hospitals should combine themselves and make full use of the integration of wechat and other information media with medical and health information, so as to become an important means to enhance the hospital brand and expand the influence of hospitals.

\subsection{Analysis of functional attributes of smart clinic}

\subsubsection{Must-be quality service indicators}

The must-be quality are the basic quality requirements that users believe must be met. Users will not show great satisfaction when such needs are met, but it will be extremely dissatisfied if such needs are not met. According to the survey results, there is no necessary attribute for patients to evaluate the functions of smart clinic, which may be due to the fact that the smart clinic in Tianjin is still in its infancy. Compared with the traditional medical treatment mode, the popularity of smart outpatient service is not high, especially for patients from other places, the understanding of smart outpatient service is low, and it is not considered as a necessary function. Therefore, patients' understanding of the construction of smart clinic should be strengthened. The hospital can carry out all-round, three-dimensional and multichannel comprehensive publicity of smart clinic in combination with new media ${ }^{[5]}$, such as setting up publicity posters and operating machines to introduce various functions of smart clinic. Secondly, in the clinic department of the hospital, there are some middle-aged and elderly patients. It is difficult for them to understand the operation of smart clinics, and they are not familiar with the operation process. Therefore,they hold that such smart clinics is not necessarily set in the medical treatment process. Therefore, the design of smart clinics system should also be simplified in its operation process as much as possible to adapt to different age groups, especially for elderly patients or people with low learning ability, and the intelligent voice guidance function should be added ${ }^{[6-8]}$. At the same time, potential target groups should be sought for key training. Patients or their families aged between 20 and 40 are the biggest target customers, which can be guided and preached $^{[9]}$.

\subsubsection{One-dimensional quality service indicators}

One-dimensional quality is the service that users expect to provide, and it has a linear relationship with satisfaction. The increase of one-dimensional quality will improve the satisfaction of users; otherwise, it will cause dissatisfaction of users. In 14 indicators, 3 items belong to the one-dimensional quality, are: (1) Orderliness in waiting for calling for diagnosis:Maintaining the order of medical treatment through smart outpatient service and supporting functions such as initial diagnosis, follow-up and check-in can greatly improve the efficiency and order of medical treatment and improve patient satisfaction ${ }^{[10]}$. Failure to maintain the order of medical treatment will greatly affect patients' medical experience. (2) Security of multiple payment methods: The online payment function in smart clinics can avoid the queuing for payment, and the time for medical treatment can be saved ${ }^{[11]}$, which greatly improve patient satisfaction. Therefore, based on ensuring the security of online payment, the clinics department needs to formulate corresponding plans to solve the problems such as overpayment and missed payment in time. (3) Accuracy of intelligent drug dispensing: Intelligent automatic drug dispensing system can realizes intelligent drug management,and patients can to quickly complete drug collection ${ }^{[12]}$. The accuracy of drug dispensing is the most concerned issue for hospitals and patients. However, the intelligent drug dispensing system has not been introduced to China for a long time, and its functions have not yet been perfected. The equipment cannot fully and accurately identify the drug dosage form and packaging, and in special cases, pharmacists need to manually adjust ${ }^{[13]}$. Therefore, its current application scope is relatively limited, and there is still a large space for improvement.

\subsubsection{Attractive quality service indicators}

The satisfaction of the attractive quality will give surprise to the user, and the absence will not be dissatisfied. Therefore, attention should be paid to improving the attractive quality while meeting the basic needs and service expectations of patients. In this survey, the item of a attractive quality is $79 \%$, and most of the indicators of patient satisfaction are greater than 0.7 , indicating that the service function has been recognized by patients, which can be maintained and improved sustainably. First of all, the time accuracy and convenience of appointment registration can be 
carefully divided into appointment periods to ensure that each patient can see a doctor on time. Unified management of appointment number source ${ }^{[14]}$ can ensure orderly medical treatment of patients, improve the efficiency of appointment, solve the problem of "three long and one short", and improve patient satisfaction. Secondly, it is the manual guidance and convenience of operation of multi-functional self-service. Patients are instructed to use the multi-functional self-service function, and special personnel are set to guide the operation of the equipment, maintain and manage it, and ensure its normal use. It gradually becomes a necessary intelligent device ${ }^{[15-16]}$. Thirdly, the implementation of intelligent guidance is relatively simple in application and convenient in operation, which can optimize the medical experience ${ }^{[17]}$. However, the guidance mode is relatively standardized and does not include enough symptom terms, which needs to be supplemented to improve the intelligent guidance function. Finally, wechat platform is rich and close to the people. It has become a popular trend for hospitals to use wechat public platform for health education. During the COVID-19 outbreak, mobile phone software has been widely used for health education. At the same time, there are some problems, such as most of the health information is uniform, and there is no analysis of the characteristics of hospitals and patients ${ }^{[18-19]}$. Therefore, hospitals should pay attention to patients' concerns, provide wonderful short information, easy to understand language, and improve patients' feelings of use.

\subsection{Better-Worse quadrant chart analysis of smart clinic service demand}

Based on analysis of importance and satisfaction quadrant diagram in figure 2, There are three items in the "advantage area" in FIG. 2, that is, the satisfaction and importance of users are both high, indicating that the work is mainly focused on this area. The main tasks are the ordering of waiting and calling numbers, ensuring the safety of online payment and ensuring the accuracy of intelligent dispensing, so as to meet the above functions and maintain patient satisfaction and the advantage of intelligent outpatient service. The other 11 items are in the "retention zone" of Figure 2, that is, patient satisfaction is high, but importance is not high, but these functions still improve patient satisfaction and therefore need to be maintained. Users' demand attributes are periodic and not invariable ${ }^{[20]}$. With the passing of time and the continuous improvement of function, patients will gradually adapt to the attractive quality intelligent clinic without surprise, which will gradually even become the basic quality. The change of attractive quality will be an inevitable trend. Therefore, in accordance with the principle of "ensuring the must-be quality, strengthening the desired attributes, and focusing on the attractive attributes", the hospital is required to always excavates the potential needs of patients to update the service functions of intelligent outpatient service and provide high-quality services.

\section{Conclusion}

The application of smart clinic service in Tianjin Third-class A Hospital needs to be further popularized. The service functions such as wechat platform information push, waiting for calling for diagnosis, multiple payment methods and intelligent drug dispensing need to be improved and perfected. According to the results of Kano, the hospital management department can give priority to popularizing the application of smart clinic service through various publicity means, and continue to maintain the service items with high patient satisfaction so as to promote the efficient combination of "Internet + medical health". As a result, it can promote the development of China's medical and health undertakings.

\section{Acknowledgments}

This article is suppoprted by Project of Tianjin Association of Science and Technology (TJSKXJCZX201917).

\section{References}

[1] General Office of the State Council. Opinions on promoting the development of "health" Internet + [EB/OL]. [201804-28]. http://www. gov. cn/zhengce/content/2018-04/28/content_5286645. htm [2020-01-05]

[2] Liu Aijun, Wang Tao. Discussion on the necessity of intelligent management and coordination system for the construction of intelligent clinics department[J]. Chinese Hospitals,2020,24(09):51-52.

[3] Liu Qizhen, Wang Hongxia. Analysis on the construction of intelligent clinics service in grade a hospitals [J]. Journal of Medical Informatics,2018,39(06):26-29+33.

[4] Wang Jingjun, Xing Hua. Research on the influencing factors of Internet medical user satisfaction based on Kano model [J]. Shanghai Medical \& Pharmaceutical Journal,2018,39(17):51-55.

[5] Wu Minhua. The feasibility analysis of Kano model in the survey of clinics service [J]. Journal of Traditional Chinese Medicine Management,2020,28(17):159-161.

[6] Yuan Yuan, Beishan Qiuxiong, Hu Junfei, et al. Analysis of community elderly tele-care service demand based on 
Kano Model [J]. Journal of Nursing Science,2020,35(01):5-9.

[7] Chen Yuhui, Tian Mengjia. Research on the development status and countermeasures of intelligent outpatient service based on health and medical big data -- A case study of Hefei High-tech Cardiovascular Hospital[J]. Jiangsu science and technology information,2020,37(10):21-24.

[8] Zhang Ru Research on hospital publicity in the era of all media[J]. Chinese Medical Humanities,2017,3(05):18-21.

[9] Wang Rong, Zhang Chunmei, Tang Xiaomin, et al. SWOT analysis of cloud outpatient department in a class a hospital in zhejiang province[J]. Medicine and Society,2017,30(11):68-71.

[10] Wu Liang. Discussion on the problems and countermeasures of orderly treatment in modern hospital patients [J]. $J i$ angsu Health System Management,2017,28(01):41-42.

[11] Zou Qingsheng, Chen Chuntao, Meng Zuowei, et al. Building smart hospital and improving online payment ratio of outpatient service [J]. China Quality,2019(11):87-89.

[12] Yu Wenlin, Xia Ping, Zhang Yuanyuan. Research on the willingness of outpatients to use medical mobile payment in public hospitals in Guangzhou[J]. Medicine and Society,2020,33(03):83-87.

[13] Zhang Jing, Zhou Zheng, Zhao Rui. Optimization of intelligent Dispensing System in Outpatient Pharmacy of Our hospital[J]. Chinese Journal of Modern Applied Pharmacy,2017,34(10):1485-1488.

[14] Chen Boxun, LI Jian, Zhou Yansheng, et al. Design and Application of intelligent booking platform for hospital inspection [J]. Medicine and Society, 2016,32(09):106-108.

[15] Xiong Lin. et al. The application of self-service terminals in nursing management[J]. Journal of Traditional Chinese Medicine Management,2019,27(14):70-71.

[16] Chen Jiaju, Liu Yang, Chen Jianyi, et al. Design and implementation of intelligent self-service platform in general hospital based on "Internet +" [J]. China Medical Education Technology,2020,34(05):646-650.

[17] Zhu Jing, Song Zhijian, Song Xia. The application effect of intelligent guidance system in outpatient department [J]. Smart Healthcare, 2020,6(18):7-9.

[18] Wan Yingli, Hou Xiaorong. Research on information service of hospital wechat public platform [J]. Journal of Medical Informatics, 2018,39(01):64-69.

[19] Wang Lu, Sheng Jiaqi, Zhang Lu, et al. Study on user needs of functional attributes of Hangzhou "smart medical care" system[J]. Chinese Journal of Ergonomics,2017,23(03):35-39.

[20] Kano N. Life cycle and creation of attractivequality[R]. The 4th International QMOD Quality Management and Organizational Development Conference. Sweden: Linkoping University,2001. 